192 WIDE AWAKE SURGERY USING LOW VOLUME PHRENIC NERVE SPARING INTERSCALENE AND LOW VOLUME SUPERFICIAL CERVICAL PLEXUS BLOCK FOR CLAVICLE NON-UNION WITH PLEXOPATHY

AP Hall* ${ }^{*}$ Z Hamoodi, I Singh, CY Ng, MD Hulgur. Wrightington Hospital, Wigan, UK

10.1136/rapm-2021-ESRA.192

Background and Aims Severe respiratory disease presents a challenge for anaesthesia in shoulder surgery. Awake surgery under interscalene block may be considered. However, the reduction in FEV1 and FVC due to phrenic nerve palsy may be prohibitive. Numerous techniques have been described as phrenic nerve sparing for shoulder analgesia. However, there is limited published data regarding effectiveness in anaesthesia for awake shoulder and clavicular surgery.

Methods We describe a case of a 64-year-old male undergoing brachial plexus exploration with open reduction and internal fixation for clavicle non-union. Following an injury 10 months previously, he presented with medial cord symptoms; sensory deficit predominately in the ulnar nerve distribution and hand weakness. Significant comorbidities included severe COPD with a FEV1 $<27 \%$.

A low volume phrenic nerve sparing interscalene block with superficial cervical plexus block $(5 \mathrm{ml}$ of $0.5 \%$ Bupivaicaine with Adrenaline for each) was performed under ultrasound guidance. The skin and subcutaneous layers were infiltrated with $10 \mathrm{ml}$ of $1 \%$ Xylocaine with Adrenaline as a field block.

Results Using a direct approach to the clavicle, the non-union was excised and fracture ends debrided. The inferior periosteum was carefully elevated off the clavicle to protect the brachial plexus. There was an intraoperative improvement in ulnar nerve symptoms observed when the lateral clavicle was reduced. There was no evidence of clinically significant phrenic nerve involvement; oxygen saturations were stable and the patient remained comfortable with no respiratory symptoms.

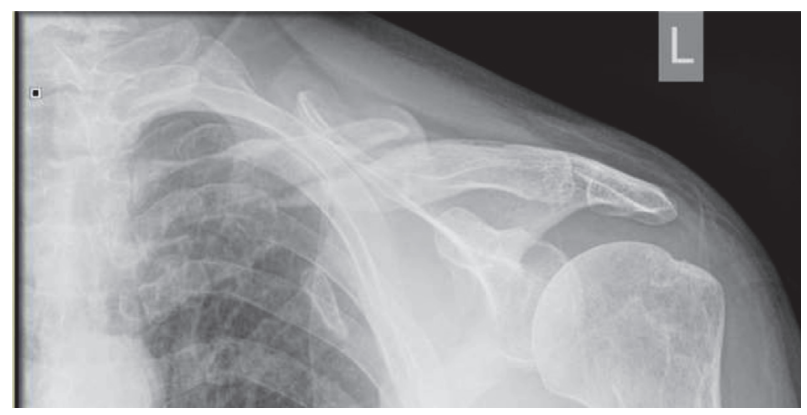

Abstract 192 Figure 1

Conclusions Low volume regional anaesthetic techniques can facilitate awake shoulder and clavicular surgery without clinically significant phrenic nerve involvement in selected patients.

\section{SUPRA-INGUINAL ILIAC FASCIA BLOCK AS SOLE ANESTHETIC TECHNIQUE FOR FEMORAL NAILING SURGERY}

F Teixeira*, D Gonçalves, C Peixoto De Sousa, AP Pereira, C Santos. Centro Hospitalar de Trás-os-Montes e Alto Douro, Vila Real, Portugal

10.1136/rapm-2021-ESRA. 193
Background and Aims Regional anesthesia, which includes peripheral nerve blocks, contributes to a multimodal analgesic approach. Iliac fascia block is an alternative or complementary analgesic technique for knee, thigh and hip surgery.

Methods 93-year-old man, ASA IV, with history of hypocoagulated atrial fibrillation, NYHA Class III heart failure, benign prostatic hypertrophy, hypertension, diabetes, dementia and dyslipidemia scheduled for femoral intramedullary nailing.

Given the patient's comorbidities and general condition, with his possible intolerance to the hemodynamic rebound produced by general or spinal anesthesia, the team decided to resort to iliac fascia block as an anesthetic technique.

Caregiver's consent to anesthesia was obtained.

To perform the block, a suprainguinal ecoguided approach was used. A linear probe was placed immediately below the antero-superior iliac spine (ASIS). The ASIS was identified and the probe placed transversely and rotated towards the umbilicus. An out of plane technique and a $50 \mathrm{~mm}$ needle were used and $20 \mathrm{~mL}$ of $1.5 \%$ mepivacaine and $20 \mathrm{~mL}$ of $0.375 \%$ ropivacaine were injected into the fascial illiaca.

The block was tested after 40, with loss of sensitivity and muscle strength in the area of the LCFN and FN. The surgery began 50' after the block. 15' and 25' into surgery, 30 $\mathrm{mg}$ and $70 \mathrm{mg}$ of propofol were administered, respectively, due to patient agitation.

Results The surgery lasted 60' and there were no complications or episodes of hemodynamic instability.

Conclusions The anesthetic approach used may be another option in hemodynamically unstable patients in which the most common anesthetic techniques incur an important hemodynamic rebound.

\section{THE EFFECT OF FEMORAL NERVE BLOCK VERSUS ADDUCTOR CANAL BLOCK ON THE QUALITY OF EARLY RECOVERY AFTER REVISION TOTAL KNEE ARTHROPLASTY, A RETROSPECTIVE STUDY}

A AlJa'bari*. DMF Hospital, Riyadh, Saudi Arabia

\subsection{6/rapm-2021-ESRA. 194}

Background and Aims Multimodal pain analgesia strategies are common in perioperative management of total knee arthroplasty (TKA), although the role of adductor canal blocks (ACB) versus femoral nerve block on early postoperative recovery for revision knee surgery is not investigated. The purpose of this study is to independently evaluate the effect of ACB on short-term postoperative outcomes including (1) length of stay (LOS), (2) postoperative narcotic utilization, and (3) function with physical therapy in revision TKA.

Methods We retrospectively identified a cohort study of consecutive 40 patients from January 2019 to July 2019 who had undergone unilateral revision TKA using a single-shot ACB (19 patients) vs femoral nerve block (21 patients) under spinal anesthesia ( hyperbaric 0.5\% Marcaine $2.5 \mathrm{ml}$ and 20 microgram fentanyl) in addition to a standardized multimodal pain analgesia protocol. These 2 groups were compared using independent sample t-tests with primary end points of interest being distance ambulated after surgery, and inpatient narcotic use.

Results Quadriceps strength was better preserved in adductor group than in femoral group. Walking meters and going 